\title{
III. Über Yttrocrasit, ein neues Yttrium-Thorium- Urantitanat.
}

\author{
Von \\ W. E. Hidden und C. H. Warren in Boston, Mass. \\ Ubersetzt von B. GoBnor.
}

Der Krystall, der das Material für die Analyse lieferte, wurde vor drei Jahren im Burnet County, Texas, von Herrn J. J. Barringer gefunden, der auch das berühmte Gadolinitvorkommen unmittelbąr am Coloradostrome in Llano County, jetzt unter dem Namen Barringer Hill bekannt, entdeckte. Er fand sich unter dem Gesteinsschutt, der bei einer Schürfung nach Gadolinit herausgeworfen worden war und zeichnete sich durch seine Größe vor mehreren Stücken im losen pegmatitischen Material aus. Der Fundort liegt üstlich gegenüber dem Barringer Hill, ungefähr drei Meilen davon entfernt, in einem Gebiete des Burnet County, wo körniger Granit und Pegmatit in großen Mengen sich vorfindet. Der Krystall war vollstïndig, abgesehen vom einen Ende und wog, wie er gefunden wurde, ca. 60 g. Er zeigle rhombische Symmetrie; aber die Flächen erwiesen sich zu uncben für cine befriedigende Messung. Der Habitus ähnelte sehr einer in Danas Mineralogie S. 738 für Yttrotantalit angegebenen Figur. Es waren die drei Pinakoide, das primüre Prisma und ein Makrodoma zu sehen; die Basis herrschte vor.

Der Krystall war mit einer dünnen Schicht von braunem, undurchsichtigem, amorphem Material überzogen. Es war dies ohne Zweifel ein wasserhaltiges Umwandlungsproduct, sehr ähnlich dem gelblichbraunem Überzuge, den man am Polykras (?) von Nord- und Süd-Carolina wahrnehmen kann. Das frische Material im Inneren des Krystalles erscheint von schwarzer Farbe, zeigt lebhaften Pech- und IIarzglanz, nicht unähnlich dem des Polykras und Euxenit, und hat, wie diese beiden Mineralien, einen unchenen und schwach muscheligen Bruch. Die Ilärte ist $5, \mathbf{5}-6$. 
Unter dem Mikroskope erscheint an Bruchstücken durch die Kanten cine bernstein- bis hellgelbe Farbe. Sehr dünne Partien sind fast farblos und durchsichlig. Nahe der Oberfläche erkennt man stellenweise kleine schwarze Flecken; sie sind in der Grundmasse zerstreut und vermutlich Umwandlungsproducte oder mechanische Einschlüsse. Zwischen gekreuzten Nicols sieht man, daß das Mineral ein Gemenge eines isotropen und eines schwach doppeltbrechenden Materials darstellt. Stellenweise ist deutlich sphärolithische Structur bei starker Vergrößerung zu erkennen; sonst lüßt sich etwas sicheres über die optischen Eigenschaften nicht aussagen. Das Mineral ist also nicht streng homogen. Diese Tatsache, zugleich in Hinsicht auf den Gehalt an Wasser und Kohlendioxyd, läßt vermuten, daß das Mineral ein wasserhaltiges Umwandlungsproduct eines anderen ursprünglich wasserfreien Minerals ist. Es ist zu erwähnen, daß eine solch inhomogene Structur auch für den Polykras (?) von Nord- und Süd-Carolina charakteristisch ist.

Vor dem Lötrohre schmilzt das Mineral nicht, nimmt vielmehr eine dunkle grauliche Farbe an und wird etwas rissig. Im geschlossenen Rohre decrepitiert es schwach und gibt etwas unterhalb der Rolglut Wasser und Kohlensäure ab. Dic Reactionen mit Schmelzmitteln am Platindrahte sind nicht charakteristisch. Das Mineral wird leicht durch Flußsäure zcrsetzt und gibt dabei einen hellgrünlichen Rückstand von Flluoriden der Erden. Fein gepulvert lüst es sich unler schwachem Aufbrausen in heißer concentrierter Schwefelsüure und gibt eine schwach opalescierende, blaß gelblichgrüne Lüsung. Fügt man dazu Salzsïure und Zink, so nimmt die Lüsung zuerst violette, dann nach und nach blaugraue und schließlich tiefblaue Fürbung an. Im inschlusse hieran sei erwähnt, daß der Polykras von Süd-Carolina in feingepulvertem Zustande in heißer concentrierter Schwefelsäure sich ohne Rückstand zu einer vollständig klaren grünen Flüssigkeit lüst.

Zur Analyse kam jener Teil des Krystalles zur Anwendung, der unter dem Mikroskope praklisch nichts von den erwähnten dunklen Flecken erkennen ließ.

Das spec. Gewicht ist 4,8043 bei $17^{\circ} \mathrm{C}$. (Miltel aus zwei sorgfältigen Beslimmungen).

Die Resultate der chemischen Analyse (Warren) befinden sich auf S. 20 oben.

IIinsichtlich der analytischen Methode sei folgendes erwähnt: Wasser und Kohlendioxyd wurden nach der Verbrennungsmethode durch direcles Glühen und Aufsaugen in conc. $\mathrm{SO}_{4} I_{2}$, bezw. Kalilauge beslimmt; die Substanz kam im Platinschiffchen in ein schwer schmelzbares Glasrohr, das nach der Vorschrift von Penfield mit Platinfolie angefüllt war. $\mathbf{A n}$ 
W. E. Ilidden und E. H. Warren. L̈bersetzt von B. Goßner.

\begin{tabular}{|c|c|c|c|c|c|c|}
\hline Oxyde: & $\%$ & Mol.-Gew.: & Verhältnis: & & & \\
\hline $\mathrm{TiO}_{2}$ & 49,72 & $\div 80,15$ & $=0,620$ & \multirow{7}{*}{0,645} & \multirow{7}{*}{16,12} & \\
\hline $\mathrm{Nb}_{2} \mathrm{O}_{\mathrm{i}}$ & chgewiesen & & & & & \\
\hline $\mathrm{Ta}_{2} \mathrm{O}_{5}$ & Spur & & & & & \\
\hline$W O_{3}$ & 1,87 & $\div 332,8$ & $=0,008$ & & & 1 \\
\hline$U O_{\mathbf{3}}$ & $0,6 / 4$ & $\div 288,0$ & $=0,002$ & & & \\
\hline $\mathrm{SiO}_{2}$ & Spur & & & & & \\
\hline $\mathrm{CO}_{2}$ & 0,68 & $\therefore 44,0$ & $=0,015$ & & & \\
\hline$(Y, E r)_{2} O_{3}$ & 25,67 & $\div 268,4$ & $=0,096$ & \multirow{3}{*}{0,113} & \multirow{3}{*}{2,82} & \\
\hline $\mathrm{Ce}_{2} \mathrm{O}_{3}$ usw. & 2,92 & $\div 331,0$ & $=0,008$ & & & \\
\hline $\mathrm{He}_{2} \mathrm{O}_{3}$ & 1,44 & $\div 160,0$ & $=0,009$ & & & \\
\hline $\mathrm{Th}_{h} \mathrm{O}_{2}$ & 8,75 & $\div 268,6$ & $=0,033$ & \multirow{2}{*}{0,040} & \multirow{2}{*}{1,00} & \\
\hline $\mathrm{UO}_{2}$ & 1,98 & $\div 271,6$ & $=0,007$ & & & \\
\hline $\mathrm{PbO}$ & 0,48 & $\therefore 222,9$ & $=0,002$ & \multirow{3}{*}{0,036} & \multirow{3}{*}{0,90} & \\
\hline $\mathrm{MnO}$ & 0,13 & $\div 71,0$ & $=0,001$ & & & \\
\hline $\mathrm{CaO}$ & 1,83 & $\div \quad$ ö 6,0 & $=0,033$ & & & \\
\hline $\mathrm{MgO}$ & Spur & & & \multirow{3}{*}{0,242} & \multirow{3}{*}{6,00} & \\
\hline $\mathrm{H}_{2} \mathrm{O}$ & 4,36 & $\div 18,0$ & $=0,242$ & & & \\
\hline \multicolumn{2}{|c|}{$\mathrm{II}_{2} \mathrm{O}$ hygrosk. 0,10} & & & & & \\
\hline Summe & 00,57 & & & & & \\
\hline
\end{tabular}

einigen blinden Bestimmungen war vorher die Brauchbarkeit des Apparates erprobt worden. Die Summe aus Wasser + Kohlendioxyd ist etwas größer $(0,15 \%)$ als der Glühverlust des Schiffchens mit Inhalt, wahrscheinlich eine Folge der Oxydation von $\mathrm{CO}_{2}$. Die gute Übereinstimmung deutet darauf hin, daß wenig oder gar kein Helium oder Stickstoff im Mineral enthalten ist; nachzuweisen waren beide nicht. Nach dem Glühen halte des Mineral eine hellgelbe Farbe und löste sich schwieriger als vor dem Glühen.

Die Prüfung auf Ferroeisen nach der Methode von Penfield ${ }^{1}$ ) ergab dessen vollständige Abwesenheit. Das Verhalten der kalten schwefelsauren Lüsung gegen Kaliumpermanganat wurde demnach so gedeutet, dả $ß$ wenigstens ein T'eil des Urans in der Form von Dioxyd anwesend ist; die hierfür angegebene Zahl in der Tabelle wurde durch Titration mit Permanganat erhalten.

Für die Hauptanalyse wurde das Mineral mit Flußsäure zersetzt (nach der Methode von J. Lawrence Smith). Von dem grünlichweißen Rückstande der Fluoride der Erden wurde abfiltriert; sie wurden in die Sulfate (etwas Bleisulfat wurde hierbei ermiltelt) verwandelt und zweimal mit Ammoniak gefillt, um die Erden usw. vom Kalk zu trennen. Vom Eisen

1) Brush und Penfield, Determinative Mineralog:; S. 87. 
und Uran wurden die Erden durch Fällen mit Oxalsäure geschieden. Thorium und Cererden wurden dann nach Hillebrand ${ }^{1}$ abgeschieden und die Cererden von Thorium mit Natriumthiosulfat und Ammoniumoxalat getrennt 2). Die Erden wurden schlieBlich vor dem Glïhen und Wägen als Oxalate gefällt. Große Sorgfalt wurde auf die vollständige Abscheidung der Erdoxalate aus den Filtraten verwendet. Einige wurden fast unveränderlich wieder erhalten, wodurch aufs neue auf die von llillebrand erwähnte Vorsicht hingewiesen wird. Die Oxyde der Yttriumerden waren von hellgelber Farbe und gaben mit Salpetersäure eine blaßrote Iıösung. lhre spectroskopische Prüfung ließ das charakteristische Absorptionsspectrum des Erbiums crkennen. Das Molekulargewicht wurde zu 268,4 $\left(R_{2} O_{3}\right)$ gefunden.

Die Ceroxyde waren braun gefärbt und lüsten sich sich in Salzsäure mit gelber Farhe. Die spectroskopische Prüfung zeigte die Didymbande und ein schwaches Band in der Iage der am meisten charakteristischen Erbiumlinie, was auf eine kleine Verunreinigung hindeutete. Jas Molekulargewicht 331,0 $\left(\mathrm{R}_{2} \mathrm{O}_{3}\right)$ ist wahrschcinlich nicht genau infolge des geringen Gesamtgewichtes der 0xyde. Um in diesem Teile Eisen vom Uran zu trennen, wurde Schwefelwasserstoff durch die warme, an Ammoncarbonat nahezu gesätligte Jösung geleitel ${ }^{3}$ ).

l)as bej der Behandlung mit Flußsïure erhaltene Fillral wurde zur 'Trockne verdampft und mil Schwefelsäure abgeraucht. Dic abgekühlte Masse wurde in Wasser gelösl; mit $\Lambda$ mmoniak und frisch bercitclem farblosem $\Lambda$ mmoniumsulfid wurden dann die Hydroxyde gefïllt und noch einige Zeit auf dem Wasserbade digeriert. Die Fïllung wurde wicderholt und in den gesimlen Jiltraten dann das Wolfram bestimml. Zinn konnte nicht nachgewiessn werden. Elwas Wolfram bleibt ohne Zweifel bei Titan usw.; aber nach unserer lirfahrung hat die eben beschriebene Methode weniger Fehler im Gefolge, als die Schmelzung mit einem Gemenge von Natriumcarljonal und Nittriumsulfid. Nach der zweiten Behandlung mit Ammonsulfid blielen die gefülllen Ifydroxyde längere Zeit mit conc. Schwefelsüure in Berührung, wobei das schwarze Eisensulfid und elwas Tilan und Uran in Lösung ging. Wir lüsten die Ilydroxyde nochmals in Flußsäure, verjaglen die letztere mit Schwefelsiure, neutralisierten dann mit Ammoniak und fü̈rlen einen Überschuß von Ammoniumcarbonal und etwas farbloses Ammoniumsulfid hinzu. Auf diese Weise blicb Uran mil etwas litan in rüsung. Nach wiederholter Fïllung bei Gegenwart von Ammoniumcarbonat

1) Amer. Journ. of Sc. $1902,13,148$.

2) Siche Mel\%ger, Am. Chem. Journ. 1901, 94, 901.

3) Diese befriedigende Trennungsmethorle fur Eisen und lan rerdanken wir Herrn B. B. Bolt wood in New Haven. 
blieben beim Uran nur noch Spuren von Eisen und Titan. Vom Hauptniederschlage wurde mittels schwefeliger Säure noch etwas Eisen gewonnen. Die geringe Titanmenge, welche sich mit dem Eisen lüst, kann leicht wieder mit Nalriumacetat bei Anwesenheit von Essigsïure und schwefeliger Säure gefüllt werden.

Die geglühten Oxyde von Titan und Niob gehen nach dem Schmelzen mit Kaliumbisulfat beim Behandeln mit kaltem Wasser fast ohne Rückstand in Lüsung (von $0,3139 \mathrm{~g}$ blieben nur 0,0021 $\mathrm{g}$ ungelöst). Quanlitalive Prüfung nach der Melhode von A. A. Noyes ${ }^{1}$ ) ergab die $\Lambda$ nwesenheit von Niob; die Lüsung fürbte sich bei der Reduction mit einer Zinksäule braun; mit Quecksilberchloridlösung entstand sofort reichlich ein weißer Niederschlag. J)iese Reaction ist sehr scharf, man erhäl sie noch, wenn nur einige Milligramm Niobsäure anwesend sind. Dieser Umstand, sowie die 'Talsache, daß beim Ausziehen der Schmelze mit Wasser nur ein kleiner Rückstand blieb, deuten darauf hin, daß Niob und Tantal nur in geringen Mlengen vorhanden sind. Nach den Resultaten der folgenden Abhandlung über die Bestimmung von Niob und Tantal bei Gegenwart von Titansäure können ganz leicht mehrere Procente Niob und l'antal anwesend sein und doch kann in Wirklichkeit kein Rückstand bleiben, wenn man die Kaliumbisulfatschmelze mit Wasser auszieht. Gegenwärtig scheint eine genaue Bestimmung der kleinen Niobmenge nicht möglich zu sein. Tantal läßt sich nur in Spuren nachweisen.

Unter der Voraussetzung, daß der Niobgehalt zu klein ist, um das Molekularverhältnis nennenswert zu beeinflussen, erhält man, entsprechend obiger Tabelle, wenn man die sauren und basischen Radicale zusammenfaßt, folgende annähernde Verhältnisse:

$$
\begin{aligned}
& \mathrm{H}_{2} \mathrm{O}: \stackrel{\mathrm{II}}{\mathrm{R} O}: \stackrel{\mathrm{III}}{\mathrm{R}_{2}} \mathrm{O}_{3}: \stackrel{\text { IV }}{\mathrm{R} \mathrm{O}_{2}: \mathrm{TiO}_{2}} \text { usw. } \\
& 6: 1: 3: 1: 16
\end{aligned}
$$

$\stackrel{\text { II }}{R O}$ ist vorwiegend Kalk, $\stackrel{\text { III }}{\mathrm{R}_{2} \mathrm{O}_{3}}$ Yttriumerden und $\stackrel{\text { IV }}{\mathrm{RO}} \mathrm{O}_{2}$ hauptsächlich Thoriumoxyd. Das Mineral ist also im wesentlichen ein wasserhaltiges Titanat von Thorium und den Yttererden. Die obigen Verhältnisse können nicht mehr als ein zufälliges Zusammentreffen sein; aber sie sind hinreichend scharf, um zu Gunsten der Richtigkeit unserer Annahme zu sprechen. Angesichts der Unrollkommenheit der $\Lambda$ nalyse und unserer wenig cingehenden Kenntnisse über die wahren Molekularverhältnisse der seltenen Erden und sauren liadicale lohnen sich eingehendere Erörterungen über die Constitution unseres Minerals nicht. Bevor wir überhaupt eine richlige Auffassung von der ganzen Grupje der sog. Titano-Niobate bekommen wollen, müssen wir nicht nur bessere analytische Methoden, sondern auch

1) A System of Qualitative Analysis, Technology Quarlerly 1904, 17, No. 3. 
einen klareren Einblick besitzen, inwieweit diese Elemente und ihre Verbindungen zur Bildung isomorpher Mischungen und fester Lösungen befähigt sind.

Radioactive Eigenschaften. Iferr Prof. B. B. Boltwood war so gefällig, einige Bruchstücke des Minerales auf Radioactivität zu prüfen. Danach entspricht die gesamte Activität einem Gehalte von 10\% Thorium und 2,08\% Uran. Bei der Analyse fanden wir 2,29\% Uran und 8,7 $3 \%$ Thoriumoxyd, was mit Boltwoods Zahlen sehr befriedigend stimmt.

Das Mineral ist ohne Zweifel eine neue Species, wir wollen ihm den Namen Yttrocrasit geben. 